\title{
EVALUATION OF THE INCIDENCE AND CLINICAL CHARACTERISTICS OF GLUCOSE METABOLISM ALTERATIONS DURING THE FOLLOW-UP OF SURGICALLY TREATED INSULINOMAS
}

João Sérgio Neves, Eva Lau, Joana Oliveira, Ana Isabel Oliveira, Paula Freitas, Davide Carvalho

Department of Endocrinology, Diabetes and Metabolism, São João Hospital Center

Faculty of Medicine, University of Porto, Porto, Portugal

SÃO JOÃO

\section{INTRODUCTION}

The incidence of glucose metabolism alterations during the follow-up of surgically treated insulinomas is largely unknown. Our purpose was to evaluate the incidence, and the clinical characteristics, of diabetes and prediabetes in this population.

\section{METHODS}

We retrospectively analyzed the cases diagnosed as insulinomas in a Central Hospital in Portugal (Hospital São João) in the period between January 1980 and December 2015.

\section{RESULTS}

\section{Patients Presentation}

- We identified 19 patients with insulinoma $68 \%$ women

Age at onset of symptoms: 49 years

Whipple triad: $\mathbf{1 0 0 \%}$ patients

Neuroglycopenic symptoms: $100 \%$
Autonomic symptoms: $79 \%$

- No case was associated with MEN-1 syndrome

- 5 patients were treated with diazoxide preoperatively

\section{TUMOR CHARACTERISTICS}

- All tumors were solitary

- Median diameter of $1.8 \mathrm{~cm}$

- Tumor location Head: 10 tumors

Body: 4 tumors

Tail: 4 tumors

Uncinate process: 1 tumor

- Only 1 tumor, did not present positive insulin staining (with evidence of endogenous hyperinsulinism)

- There were no cases of lymphatic or vascular invasion

\section{Glucose Metabolism Alterations}

- Median follow-up: 48 months

- only 4 patients with a follow-up inferior to 6 months because of loss of follow-up or recent intervention.

9 patients (47\%) presented

\section{glucose metabolism alterations}

\section{$\rightarrow$ - 8 patients developed diabetes \\ 4 in immediate postoperative period \\ 1 patient 4 months after surgery \\ 3 patients $10-12$ years after surgery}

- 1 patient presented prediabetes (54 months

- Mean age at diagnosis: 54 years after surgery)

- Treatment 3 patients treated with insulin

3 treated with oral antidiabetic drugs

1 with insulin and oral antidiabetic drugs

2 with lifestyle intervention

- Complications: only 1 patient presented microalbuminuria (no other micro- or macrovascular complications observed)

\section{CONCLUSIONS}

Glucose metabolism alterations are a frequent complication during the follow-up of insulinomas. Prevention, early diagnosis and treatment of diabetes in patients with surgically treated insulinomas must be a priority during the follow-up of these patients. 